\title{
Desenvolvimento de protocolo para Validação e Qualificação Técnica de Visor de Hemaglutinação
}

\author{
Development of protocol for Validation and Technical Qualification of \\ Hemaglutination Display
}

\author{
Vinicius Matheus Knihs; Alexandre Geraldo²; Anna Paula de Borba Batschauer³ Marshell Ferreira \\ Almeida Ferraz ${ }^{4}$
}

\begin{abstract}
${ }^{1}$ Acadêmico de Biomedicina, da Universidade do Vale do Itajaí (UNIVALI), Campus Itajaí, SC, Brasil. E-mail: knihsvmk369@gmail.com

${ }^{2}$ Docente da Universidade do Vale do Itajaí (UNIVALI), SC, Brasil. Consultor e avaliador do Programa Nacional de Qualificação da Hemorrede (PNQH)/ Coordenação Geral de Sangue e Hemoderivados do Ministério da Saúde. E-mail: alexandregeraldo@univali.br

${ }^{3}$ Docente titular de Hematologia nos cursos de Farmácia e Biomedicina da UNIVALI, SC, Brasil. Responsável técnica do Lab. de Análises Clínicas Batschauer Ltda. E-mail: batschauer@univali.br ${ }^{4}$ Engenheiro de Projetos Seis Sigmas. E-mail: marshell@univali.br
\end{abstract}

\begin{abstract}
RESUMO: Os laboratórios de análises clínicas e bancos de sangue em todo o Brasil, realizam em sua rotina exames de tipagem sanguínea, que são observadas através de reações de hemaglutinação. Equipamentos e instrumentos são utilizados na rotina laboratorial como auxílio da conclusão da tipagem sanguínea ampliando as reações imunohematológicas, porém estes equipamentos tiveram sua produção descontinuada, dessa forma, faz-se necessária a elaboração de protocolos de validação e de qualificação, para que os mesmos possam ser fabricados corretamente. Este trabalho tem como objetivo desenvolver um protocolo para a validação e um para qualificação técnica de visores de hemaglutinação. Foram pesquisados nas plataformas de busca Google Acadêmico e PubMed 1429 artigos, sendo utilizados 155 artigos para construção do protocolo publicados entre 1/01/1990 até 01/10/2018. Após a realização da leitura dos artigos foi construído um check-list para a validação, e um para a qualificação técnica, contendo itens a serem avaliados. Estes itens receberam pesos diferentes para a pontuação final (peso 1 e peso 2), de acordo com o nível de interferência para a análise do resultado, bem como média mínima que o equipamento deve atingir ao passar pela análise. Os escores finais variam de 24 a 12 para a validação e de 22 a 11 para a qualificação. Caso esta média não seja atingida, o equipamento é desclassificado. Os protocolos de validação e qualificação técnica, redigidos na forma de check-list neste trabalho, não foram testados em visores de hemaglutinação, somente desenvolvidos com base na observação in loco destes, por isso são necessários estudos futuros com a aplicação dos protocolos. Contudo, este protocolo irá proporcionar maior segurança na emissão de resultados de tipagem sanguínea no futuro, para laboratórios que utilizam o visor de hemaglutinação.
\end{abstract}

Palavras-chave: Desenho de Equipamento, Estudos de Validação, Hemaglutinação, Inovação

\begin{abstract}
Clinical analysis laboratories and blood banks throughout Brazil, carry out blood typing tests in their routine that are observed through hemagglutination reactions. The laboratory routine uses equipment and instruments as an aid to the completion of blood typing, increasing immunohematological reactions, but this equipment has had its production discontinued, so it is necessary to develop validation and qualification protocols, so that they are properly manufactured. This article aims to develop a protocol for the validation and one for the technical qualification of hemagglutination displays. 1429 articles were searched on the Google Scholar and PubMed search platforms, using 155 articles for the construction of the protocol published between 1/1/1990 and 10/1/2018. After reading the articles, one checklist was built for validation and one for technical qualification, containing items to be evaluated. These items received different weights for the final score (weight 1 and weight 2), according to the level of interference on the observation of the result, as well as the minimum average that the equipment must reach when going through this analysis. Final scores range from 24 to 12 for validation and 22 to 11 for qualification. If the equipment does not reach this average, then it is disqualified. The validation and technical qualification protocols, written in the form of a checklist in this work, were not tested on hemagglutination displays, only developed based on their observation in loco, so future studies with the application of the protocols are necessary. However, this protocol will provide greater security in the issue of blood typing results in the future, for laboratories that use the hemagglutination display.
\end{abstract}

Keywords: Equipment Design, Hemagglutination, Innovation, Validation Studies 


\section{INTRODUÇÃO}

Os laboratórios de análises clínicas e bancos de sangue em todo o Brasil, realizam em sua rotina exames de tipagem sanguínea. Esses exames, que são observados através de reações de hemaglutinação, são realizados principalmente através da técnica em tubo. Para aumentar a sensibilidade das reações de hemaglutinação, equipamentos e instrumentos são utilizados na rotina laboratorial como auxílio da conclusão da tipagem sanguínea ampliando as reações imunohematológicas (BRASIL, 2013; BRASIL, 2014a).

As reações de hemaglutinação são extremamente importantes para laboratórios de análises clínicas e hemocentros, pois elas são as reações que permitem analisar o grupo sanguíneo de um paciente ou doador de sangue (BRASIL, 2017) Quando utilizada a técnica em tubo para a classificação do grupo sanguíneo utiliza-se um equipamento denominado "visor de hemaglutinação". O visor de hemaglutinação é um importante equipamento que está em falta hoje no mercado brasileiro, porém que é ideal para este tipo de análise (BRASIL, 2014a). Este produto era comercializado pela empresa Phoenix Luferco ${ }^{\circledR}$, porém ela abandonou sua produção, e os laboratórios de análises clínicas e hemocentros, passaram a desenvolver técnicas alternativas para a visualização desse tipo de reação (PHOENIX, 2018; VIEIRA, 2016). O visor de hemaglutinação consiste em um espelho côncavo. A reação de hemaglutinação é ampliada pelo reflexo do espelho, com auxílio de uma luz incidente para facilitar a visualização. A ampliação se faz necessária para aumentar a sensibilidade visual do teste, e diminuir a chance de resultados falsos negativos nos testes imuno-hematológicos (VIEIRA, 2016).

Novas tecnologias são de suma importância para aprimorar a visualização das reações imunohematológicas nas técnicas em tubo, visto que a produção de visores de hemaglutinação foi descontinuada, e estes equipamentos eram o "Padrão Gold" para as análises imunohematológicas (BRASIL, 2014a; VIEIRA, 2016). Essas novas tecnologias necessitam de qualificação técnica e de validação antes de serem devidamente registradas na Agência Nacional de Vigilância Sanitária (ANVISA), Ministério da Saúde e Instituto Nacional de Metrologia, Qualidade e Tecnologia (INMETRO) (BRASIL, 2011; BRASIL, 2014b; INMETRO, 2016).

A validação e qualificação técnica são procedimentos essenciais para a consolidação de qualquer produto, pois é através desse processo que o mesmo será classificado como propício ou não para comercialização (BRASIL, 2001). A qualificação pode ser definida como uma série de operações documentadas de acordo com um plano de testes predeterminados e critérios de aceitação definidos, garantindo que fornecedores, insumos, equipamentos e instrumentos atendam a requisitos especificados, ou seja, 0 conjunto de testes que permite avaliar a funcionalidade do item avaliado, se o mesmo funciona da maneira que deve ser (refere-se a instalação). Já a validação é a evidência documentada de que um procedimento, processo, sistema ou método realmente conduz aos resultados esperados, ou seja, se o item analisado proporciona a obtenção do resultado esperado (refere-se ao processo e ao resultado). Posto isso, fica claro que o processo de validação depende da qualificação e vice-e-versa (WHO, 2002). Esses processos visam a qualidade do objeto ofertado, que é garantida através das Boas Práticas de Funcionamento (BPF), que são os componentes da Garantia da Qualidade que asseguram que os serviços são ofertados com padrões de qualidade adequados, conforme legislações em vigência (BRASIL, 2011). 
É de suma importância que a qualidade e a confiabilidade de um produto sejam asseguradas, através dos processos de validação e qualificação técnica. Portanto, este trabalho teve como objetivo desenvolver um protocolo para a validação e a qualificação técnica de visores de hemaglutinação, possibilitando o seu uso para fins de rotina laboratorial.

\section{MATERIAL E MÉTODOS}

\section{Busca científica para elaboração dos protocolos}

No período de agosto de 2017 a novembro de 2018, foi iniciado o processo de consulta bibliográfica de artigos científicos para a elaboração dos protocolos. A pesquisa foi realizada em periódicos nacionais e internacionais em bases de dados com os termos "validation studies immunohematology", denominado T1, "validation protocol immunohematology" (T2), "validation methods immunohematology" (T3), "qualification methods immunohematology" (T4), "methods immunohematology" (T5), que foram utilizadas na base de dados US National Library of Medicine National Institutes of Health (PubMed $\left.^{\circledR}\right)$ (PUBMED, 2018). Os termos "protocol for validation of immunohematology techniques" (T6) e "validação de equipamentos" (T7), foram utilizados em sites de mecanismos de pesquisa ou ferramentas de busca (search engines) (GOOGLE, 2018). Os critérios para inclusão dos manuscritos foram a data, sendo selecionados aqueles publicados entre 1990 a 2018 e que na apresentação do título mostrassem estudos específicos com o tema imuno-hematologia laboratorial. Já os critérios para exclusão dos artigos, foram os repetidos ou que fugissem ao tema de estudo. Foram também utilizados livros relacionados à Hemoterapia e Imuno-hematologia e documentações da Agência Nacional de Vigilância Sanitária (ANVISA) que determinam como realizar os processos de validação e qualificação técnica de equipamentos.

Após a investigação inicial dos termos nas plataformas de busca, os mesmos foram pesquisados novamente, porém com o uso de filtros específicos. Foi utilizado na plataforma PubMed ${ }^{\circledR}$ filtro de data, que selecionou artigos publicados do período de 01/01/1990 até 01/10/2018, e no Google Acadêmico, o termo T6 foi pesquisado com resultados apenas em português, e o termo $\mathrm{T} 7$ foi pesquisado com o uso de aspas. Os itens do check-list a serem avaliados foram desenvolvidos com base nos protótipos de visores de hemaglutinação que foram produzidos pelos alunos do curso de Engenharia de Produção da UNIVALI (VIEIRA, 2016).

\section{Desenvolvimento de escore de qualificação e validação}

O check-list, foi elaborado através da determinação de um escore para cada item, no qual a pontuação foi atribuída de 0 a 2, sendo 0: Não Cumpre, 1: Cumpre Parcialmente e 2: Cumpre totalmente. Para classificar um item como validado ou qualificado, foi calculada média entre a nota máxima a ser atingida no escore e a nota mínima, de modo que, os equipamentos que atingirem essa média, ou a superarem, sejam classificados como validados e/ou qualificados (AYSOLA et al, 2017). Os visores de hemaglutinação que obtiveram média menor que a estipulada para validação ou qualificação, não podem ser utilizados na rotina laboratorial. Concomitantemente, ao desenvolvimento do escore foi desenvolvida uma pontuação que levasse em consideração critérios técnicos mínimos que 
qualquer visor de hemaglutinação devesse atingir para a garantia de resultados imunohematológicos confiáveis.

De forma a calcular a nota para que o visor fosse classificado como validado ou qualificado, foram desenvolvidas duas fórmulas, uma para a validação e outra para a qualificação, conforme apresentadas na Figura 1 e na Figura 2, respectivamente. A elaboração das fórmulas foi feita utilizando o símbolo da somatória, visto que o resultado final de ambas é a soma dos escores dos itens de peso 2 com a soma dos escores dos itens de peso 1.

Figura 1: Fórmula de cálculo da avaliação do resultado final do check-list de Validação.

$$
\sum_{i^{\prime}=(X \times 2)}^{5} i^{\prime}+\sum_{i^{\prime \prime}=(X \times 1)}^{2} i^{\prime \prime}=Y
$$

Legenda: $X=$ nota atribuída ao respectivo item; $Y=$ nota final atingida pelo visor de hemaglutinação analisado; 5 = quantidade de itens com peso 2 no check-list; 2 = quantidade de itens com peso 1 no check-list, i' = " $X$ " multiplicado pelo peso 2; i" = "X" multiplicado pelo peso 1.

Fonte: Elaborado pelo autor (2019).

Figura 2: Fórmula de cálculo da avaliação do resultado final do check-list de Qualificação.

$$
\sum_{i^{\prime}=(X \times 2)}^{2} i^{\prime}+\sum_{i^{\prime \prime}=(X \times 1)}^{7} i^{\prime \prime}=Y
$$

Legenda: $X=$ nota atribuída ao respectivo item; $Y=$ nota final atingida pelo visor de hemaglutinação analisado; 2 = quantidade de itens com peso 2 no check-list; 7 = quantidade de itens com peso 1 no check-list, i' = " $X$ " multiplicado pelo peso 2; i" = "X" multiplicado pelo peso 1 .

\section{Patentes do INPI}

Posteriormente ao desenvolvimento dos escores e do check-list, foi realizada uma pesquisa sobre registro de patentes, como os visores de hemaglutinação não estavam mais sendo fabricados e comercializados no Brasil. A pesquisa foi realizada no site do Instituto Nacional da Propriedade Industrial (INPI, 2019), a fim de verificar a possível existência de alguma patente sobre visores de hemaglutinação no Brasil.

\section{RESULTADOS}

Os documentos pesquisados para a construção dos protocolos, que foram publicados entre 1990 e 2018, estão apresentados na Tabela 1. Foram encontrados um total de 1429 artigos científicos sem o uso de filtro. Após a utilização de filtro avançado, obteve-se um total de 155 artigos que foram utilizados para o desenvolvimento do escore e modelo de check-list dos parâmetros de validação e qualificação para o visor de hemaglutinação. 
Tabela 1: Códigos dos termos de busca utilizados nos mecanismos de pesquisa, bem como o número de artigos apresentados pelas plataformas com e sem filtros.

\begin{tabular}{cccc}
\hline Código do termo & $\begin{array}{c}\text { Plataforma de } \\
\text { busca }\end{array}$ & $\begin{array}{c}\text { № absoluto de } \\
\text { artigos sem o uso } \\
\text { de filtro }\end{array}$ & $\begin{array}{c}\text { № absoluto de } \\
\text { artigos com o uso } \\
\text { de filtro }\end{array}$ \\
\hline T1 & $\begin{array}{c}\text { PubMed } \\
\text { PubMed }\end{array}$ & 20 & 0 \\
T2 & $\begin{array}{c}\text { PubMed } \\
\text { T3 }\end{array}$ & 41 & 0 \\
T4 & PubMed & 3 & 2 \\
T5 & PubMed & 1256 & 2 \\
T6 & Google acadêmico & 6 & 47 \\
T7 & Google acadêmico & 100 & $5^{*}$ \\
\hline Total & - & 1429 & 99 \\
\hline
\end{tabular}

Nota: * - Um artigo foi removido devido à duplicidade de publicação na plataforma.

Os itens que foram considerados para o check-list receberam pesos diferentes para a pontuação final (peso 1 e peso 2), levando em consideração o potencial interferente na avaliação do resultado do teste. Ou seja, os itens a serem avaliados que interferem diretamente na avaliação do resultado da reação de hemaglutinação, receberam um peso maior (peso 2), já os itens que não interferem diretamente na avaliação do resultado, receberam um peso menor (peso 1), sendo estes apresentados na Tabela 2 e na Tabela 3. Cabe ressaltar que, se o equipamento obtiver nota zero em qualquer item de peso 2, ele será automaticamente reprovado, não sendo classificado como validado ou qualificado.

Tabela 2: Check-list dos itens a serem avaliados para validação do visor de aglutinação e resultados esperados.

\begin{tabular}{|c|c|c|c|}
\hline Item de validação & Resultado Esperado & Peso & Nota \\
\hline Leitura de & Equipamento deve proporcionar a & & \\
\hline $\begin{array}{l}\text { intensidades de } \\
\text { aglutinação }\end{array}$ & $\begin{array}{c}\text { visualização de leituras positivas maiores } \\
\text { ou iguais a } 1+\end{array}$ & 2 & \\
\hline Qualidade do & Instrumento de ampliação deve & & \\
\hline $\begin{array}{l}\text { instrumento de } \\
\text { ampliação }\end{array}$ & $\begin{array}{l}\text { proporcionar uma ampliação nítida da } \\
\text { reação }\end{array}$ & 2 & \\
\hline Ângulo da luz & $\begin{array}{l}\text { Luz não deve atrapalhar a visualização da } \\
\text { aglutinação }\end{array}$ & 2 & \\
\hline $\begin{array}{l}\text { Execução da } \\
\text { higienização }\end{array}$ & $\begin{array}{c}\text { Higienização não deve de forma alguma } \\
\text { prejudicar os resultados observados no } \\
\text { aparelho }\end{array}$ & 2 & \\
\hline Estabilidade & $\begin{array}{l}\text { Equipamento deve ser estável, tendo sua } \\
\text { base nivelada, de forma a não oscilar }\end{array}$ & 1 & \\
\hline Durabilidade & $\begin{array}{l}\text { Lâmpada e instrumento de ampliação } \\
\text { devem possuir boa durabilidade }\end{array}$ & 1 & \\
\hline Intensidade da luz & $\begin{array}{c}\text { Intensidade da luz não deve interferir na } \\
\text { visualização da reação }\end{array}$ & 2 & \\
\hline
\end{tabular}


RBCTl

Tabela 3: Check-list dos itens a serem avaliados para qualificação do visor de aglutinação e resultados esperados.

\begin{tabular}{|c|c|c|c|}
\hline Item de qualificação & Resultado Esperado & Peso & Nota \\
\hline Interruptor & $\begin{array}{l}\text { Interruptor deve ter boa } \\
\text { durabilidade }\end{array}$ & 1 & \\
\hline $\begin{array}{l}\text { Mobilidade de } \\
\text { instrumento de ampliação }\end{array}$ & $\begin{array}{l}\text { Instrumento a ser utilizado para } \\
\text { ampliação da visualização deve ser } \\
\text { facilmente movido }\end{array}$ & 1 & \\
\hline $\begin{array}{l}\text { Flexibilidade/mobilidade } \\
\text { da haste da lâmpada (se } \\
\text { presente) }\end{array}$ & $\begin{array}{l}\text { Haste de suporte da lâmpada deve } \\
\text { possuir boa flexibilidade/mobilidade }\end{array}$ & 1 & \\
\hline Material & $\begin{array}{c}\text { Higienização do equipamento deve } \\
\text { ser fácil e não deve comprometer } \\
\text { sua funcionalidade }\end{array}$ & 2 & \\
\hline Ergonomia & $\begin{array}{l}\text { O equipamento deve ser leve, de } \\
\text { fácil manuseio e transporte }\end{array}$ & 1 & \\
\hline Manutenção & $\begin{array}{c}\text { Lâmpada e instrumento de } \\
\text { ampliação devem ser facilmente } \\
\text { substituíveis }\end{array}$ & 2 & \\
\hline Voltagem & $\begin{array}{l}\text { Aparelho deve ser bivolt } \\
(110 \mathrm{~V} / 220 \mathrm{~V})\end{array}$ & 1 & \\
\hline Tomada & $\begin{array}{c}\text { Tomada deve atender } \\
\text { especificações da NBR 14136/07 }\end{array}$ & 1 & \\
\hline Fio & $\begin{array}{c}\text { Fio não deve ser comprido ou curto } \\
\text { demais, para não dificultar o } \\
\text { manuseio do equipamento }\end{array}$ & 1 & \\
\hline
\end{tabular}

O laboratorista deve analisar os aspectos mencionados nos check-lists e classificar os itens com uma nota de 0 a 2 ( 0 = não cumpre; 1 = cumpre parcialmente; 2 = cumpre totalmente) na coluna "nota" da respectiva tabela. Após as notas serem atribuídas, o resultado do check-list de validação deve ser aplicado a fórmula da Figura 1, e o resultado do check-list de qualificação, na fórmula da Figura 2. Os resultados finais das equações devem obrigatoriamente obedecer aos critérios da Tabela 4, apresentada a seguir.

Tabela 4: Tabela de médias para classificação de validação e qualificação.

\begin{tabular}{|c|c|c|}
\hline \multirow[t]{2}{*}{ Validação } & Nota máxima & Nota mínima \\
\hline & 24 & 12 \\
\hline Média a ser atingida & \multicolumn{2}{|c|}{18 - Não zerando em nenhum item de peso 2} \\
\hline \multirow[t]{2}{*}{ Qualificação } & Nota máxima & Nota mínima \\
\hline & 22 & 11 \\
\hline Média a ser atingida & \multicolumn{2}{|c|}{16 - Não zerando em nenhum item de peso 2} \\
\hline
\end{tabular}

A média que deve ser atingida na validação e na qualificação ao avaliar o visor de hemaglutinação, foi calculada somando a nota máxima e a mínima possíveis para cada 
check-liste dividindo-as por 2. Foram então aplicadas as notas de cada critério das Tabelas 2 e 3 nas fórmulas, conforme apresentadas nas Figuras 1 e 2.

Para melhor elucidar as atividades e sumarizar a aplicação dos protocolos, foi desenvolvido um fluxograma (Figura 3) que irá auxiliar o laboratorista na realização da validação e da qualificação.

Figura 3: Fluxograma de procedimentos referentes a aplicação do check-list de Validação.

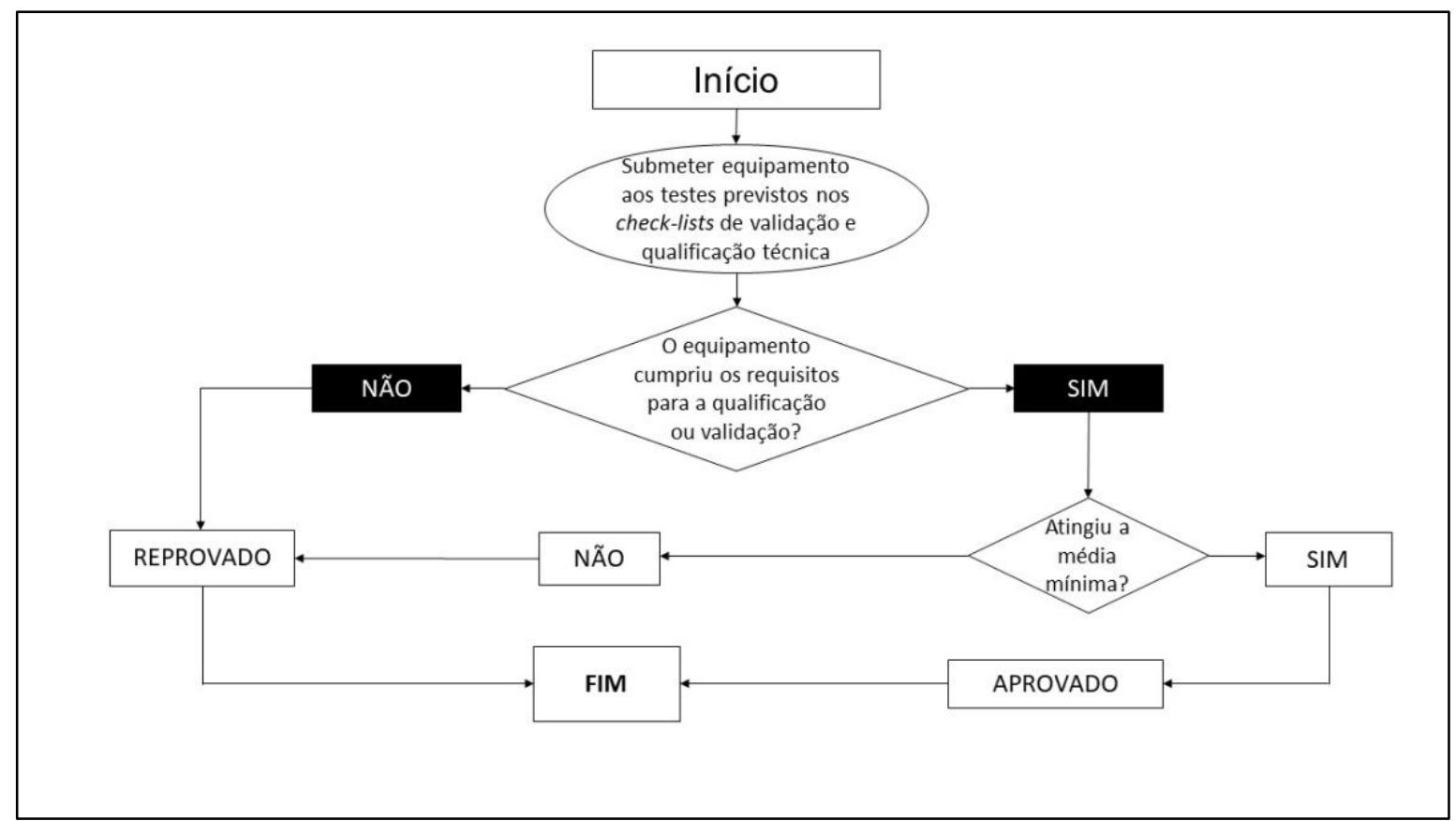

A pesquisa pela existência de patentes, feita no site do INPI, demonstrou a inexistência de patentes, que contenham os termos "visor de hemaglutinação" ou "aglutinoscópio" no seu título, ou resumo. Já para patentes que contenham quaisquer uma das palavras, foram encontrados 380 processos para "visor de hemaglutinação" no título, e 1723 no resumo, e nenhuma para "aglutinoscópio" tanto no título quanto no resumo. Ainda, para patentes que contenham palavras aproximadas a "visor de hemaglutinação" no título, foram encontradas 618 patentes, já que contenham palavras aproximadas no resumo, 2633 processos. Para patentes que contenham palavras aproximadas a "aglutinoscópio" no título, foram encontrados 38 processos, já palavras aproximadas no resumo, foram encontrados 51 processos.

\section{DISCUSSÃO}

Os processos de validação e qualificação, são de suma importância para garantir a qualidade de um produto, processo, equipamento ou serviço antes do mesmo poder ser utilizado de maneira correta e ser considerado confiável, conforme consta na BPF (BRASIL, 2011). Em um estudo realizado em 2009, fica evidente a importância da realização destes processos, visto que essa pesquisa teve como objetivo validar o analisador hematológico Sysmex XS-1000i, através da comparação entre a contagem de leucócitos do equipamento 
e a contagem manual por lâmina de extensão sanguínea. A validação classificou o produto como confiável, e isto corrobora com a importância da realização destes processos, que garantem que um item atende às expectativas que são colocadas sobre ele, diminuindo assim possíveis erros por conta de materiais calibrados erroneamente (BORGES; SIQUEIRA, 2009).

Infelizmente, foi observada uma escassez de bibliografia que fale sobre desenvolvimento de protocolos de validação e qualificação técnica de equipamentos para a área da saúde, e a maior parte das ideias para a elaboração dos resultados foram retiradas de trabalhos e artigos relacionados a outras áreas, porém que também tratam de validação e qualificação.

O desenvolvimento dos protótipos foi realizado em paralelo com o Curso de Biomedicina, com a finalidade de proporcionar a reimplantação deste produto no mercado, para fins de uso na rotina laboratorial (VIEIRA, 2016). A escolha da elaboração de um protocolo como forma de guia para a realização destes processos, se torna importante quando se observa os benefícios do emprego do mesmo, como a padronização das informações coletadas e a análise periódica dos dados obtidos (LOPES; FERREIRA; SANTOS, 2010). Uma evidência da importância da padronização e da aplicação destes protocolos foi observada em um estudo realizado em um hospital do estado de São Paulo, avaliando a conduta de tratamento empregada para pacientes com infarto agudo do miocárdio, e implementando um protocolo para a padronização da mesma. Ao fim do estudo foi observado um aumento significativo do uso de fármacos comprovadamente eficazes para o tratamento de tal enfermidade, e consequentemente uma redução significativa da mortalidade, em um período de 30 dias após a aplicação do protocolo (BORDON et al., 2004).

Em outro trabalho, foi implantado um protocolo para melhorar 0 atendimento nutricional individual na atenção primária a saúde, no município de Belo Horizonte, Minas Gerais. O estudo propôs-se uma ficha de avaliação antropométrica e um protocolo para atendimento em consultas de retorno dos pacientes estudados. Esses itens foram então implantados em nove unidades básicas de saúde e foram seguidos por seis meses, para avaliar as incongruências e necessidades de adaptação à população atendida. Após os seis meses, os protocolos foram readequados as necessidades locais, originando a versão final do documento, que melhor atendia a população, segundo o protocolo (LOPES; FERREIRA; SANTOS, 2010).

Considerando as presentes evidências, se torna nítida a importância de um protocolo a ser seguido para obter-se a validação e qualificação dos visores de hemaglutinação. Este equipamento é altamente necessário na rotina laboratorial dos testes de imunohematologia, e sua falta tem impacto direto na qualidade de trabalho de colaboradores de hemocentros e laboratórios clínicos (BRASIL, 2014a). Um estudo realizado sobre o clima organizacional do hemocentro coordenador do estado do Amapá, demonstrou que 50,3\% dos colaboradores estavam motivados por terem equipamentos necessários para a execução das tarefas diárias na instituição (BRITO, 2010). Esses resultados vêm ao encontro de outros Estados brasileiros como o do hemocentro do Sergipe, em que $48 \%$ dos colaboradores relatam que há disponibilidade de equipamentos e materiais adequados para a realização do trabalho, $49 \%$ no Hemocentro do Acre e $55 \%$ no hemocentro de Roraima (PASSOS, 2010; ROSA, 2010; MOREIRA, 2010).

O desenvolvimento e uso de protocolos que possam avaliar a validação e qualificação do visor de hemaglutinação, elaborado neste estudo poderá também 
proporcionar para futuras instituições um melhor clima organizacional, uma vez que colaboradores terão confiança em seus equipamentos de uso diário. Essas evidências demonstram que a disponibilidade de equipamentos eficientes e confiáveis no campo de trabalho reflete diretamente na eficiência dos colaboradores. A disponibilização desses equipamentos só pode ser realizada após os mesmos terem sido submetidos aos processos de validação e qualificação (BRASIL, 2017). A garantia da qualidade, independente do ramo em que se atua e deve ser considerada um mecanismo para evitar não conformidades, e, em última instância, caso ocorram, devem ser solucionados, principalmente no quesito de inserção de novos produtos no mercado brasileiro (DE PAULA; ALVES; NANTES, 2017).

\section{CONCLUSÃO}

O presente trabalho evidencia que os processos de validação e qualificação técnica são de extrema importância para classificar um equipamento como confiável, e assim, classificando-os como aptos ou não para a comercialização. Ao realizar a busca na literatura foi detectada uma escassez de artigos científicos nas plataformas pesquisadas que visam solucionar o problema proposto.

Desta forma, os protocolos de validação e qualificação apresentados como resultados do presente trabalho, em tese, estão prontos para a aplicação, tendo em vista o fato de que foram desenvolvidos somente com base na observação in loco dos visores de hemaglutinação, por isso, se faz necessário um futuro estudo no qual seja realizada a aplicação dos mesmos, e seja avaliada sua aplicabilidade na rotina laboratorial. Ressaltase também que os dados obtidos e a forma de elaboração dos protocolos, tanto o formato de check-list como a atribuição das notas, pode servir como guia para trabalhos futuros, com objetivos semelhantes, ou com estrutura semelhante para outros equipamentos na área saúde.

\section{REFERÊNCIAS}

ASSOCIAÇÃO BRASILEIRA DE NORMAS TÉCNICAS. ABNT NBR 14136: 2002 Errata 1: 2007. 2007. Disponível em: <https://www.abntcatalogo.com.br/norma.aspx?ID=58036>. Acesso em: 27 out. 2018.

AYSOLA, A.; WHEELER, L.; BROWN, R.; DENHAM, R.; COLAVECCHIA, C.; PAVENSKI, K.; KROK, E. et al. Multi-Center Evaluation of the Automated Immunohematology Instrument, the ORTHO VISION Analyzer. Lab Med., v. 48, n. 1, p. 29-38, 2017.

BORDON, J. G.; PAIVA, S. A. R.; MATSUBARA, L. S.; INOUE, R. M. T.; MATSUI, M.; GUT, A. L. et al. Redução da mortalidade após implementação de condutas consensuais em pacientes com infarto agudo do miocárdio. Arquivos brasileiros de cardiologia, v. 82, n. 4, p. 370-373, 2004.

BORGES, L. F.; SIQUEIRA, L. O. Validação de tecnologia 5diff do analisador hematológico Sysmex XS-1000i para laboratório de pequeno/médio porte. Revista Brasileira de Hematologia e Hemoterapia, v. 31, n. 4, p. 252-259, 2009. 
BRASIL(a). Ministério da Saúde. Secretaria de Atenção à Saúde. Departamento de Atenção Hospitalar e de Urgência. Imuno-hematologia laboratorial / Ministério da Saúde, Secretaria de Atenção à Saúde, Departamento de Atenção Hospitalar e de Urgência. - Brasília: Ministério da Saúde, 2014.

BRASIL(b). MINISTÉRIO DA SAÚDE.AGÊNCIA NACIONAL DE VIGILÂNCIA SANITÁRIA. Resolução - RDC no 34, de 11 de junho de 2014. Dispõe sobre as Boas Práticas no Ciclo do Sangue.

BRASIL. MINISTÉRIO DA SAÚDE. AGÊNCIA NACIONAL DE VIGILÂNCIA SANITÁRIA. Resolução - RDC no 185, de 22 de outubro de 2001. Aprova o Regulamento Técnico que consta no anexo desta Resolução, que trata do registro, alteração, revalidação e cancelamento do registro de produtos médicos na Agência Nacional de Vigilância Sanitária - ANVISA.

BRASIL. MINISTÉRIO DA SAÚDE. AGÊNCIA NACIONAL DE VIGILÂNCIAS SANITÁRIA. Resolução - RDC n 63, de 25 de novembro de 2011. Dispõe sobre os Requisitos de Boas Práticas de Funcionamento para os Serviços de Saúde.

BRASIL. MINISTÉRIO DA SAÚDE. Portaria de consolidação no 5, de 28 de setembro de 2017. Consolidação das normas sobre as ações e os serviços de saúde do sistema único de saúde.

BRASIL. Ministério da Saúde. Secretaria de Gestão do Trabalho e da Educação na Saúde. Departamento de Gestão do Trabalho na Saúde. Técnico em hemoterapia: livro texto / Ministério da Saúde, Secretaria de Gestão do Trabalho e da Educação na Saúde, Departamento de Gestão da Educação na Saúde - Brasília: Ministério da Saúde, 2013.

BRITO, J. R. S. Clima Organizacional do Hemocentro Coordenador do Estado do Amapá: enfoque no Corpo Técnico. In: Gestão de hemocentros: relatos de práticas desenvolvidas no Brasil: I curso de Especialização em gestão de hemocentros: resumos das monografias finais/ Ministério da Saúde, Secretaria de Atenção à Saúde, Departamento de Atenção Especializada. - Brasília: Ministério da Saúde, 2010.

DE PAULA, L. N.; ALVES, A. R.; NANTES, E. A. S. A importância do controle de qualidade em indústria do segmento alimentício. Revista Conhecimento Online, v. 2, p. 78-91, 2017.

INSTITUTO NACIONAL DA PROPRIEDADE INDÚSTRIAL. Guia básico de patentes. 2018. Site que fornece informações acerca da elaboração de patentes. Disponível em: <http://www.inpi.gov.br/menu-servicos/patente>. Acesso em: 24 fev. 2019.

INSTITUTO NACIONAL DE METROLOGIA, QUALIDADE E TECNOLOGIA. Documentos Necessários para Acreditação de Laboratórios de Calibração e de Ensaios segundo requisitos da NBR ISO/IEC 17025. 2016. Apresenta uma lista de documentações necessárias para acreditação de produtos, equipamentos, ou realização de ensaios de 
laboratórios de vários tipos. Disponível em:

<http://www.inmetro.gov.br/credenciamento/organismos/doc_organismos.asp?tOrganismo $=$ CalibEnsaios $>$. Acesso em: 20 set. 2017.

LOPES, A.; FERREIRA, A. D.; SANTOS, L. C. Atendimento Nutricional na Atenção Primária à Saúde: Proposição de Protocolos. Nutrição em pauta, v. 18, n. 101, p. 40-44, 2010.

MOREIRA, R. M. Clima Organizacional do Hemocentro de Roraima - Hemoraima. In: Gestão de hemocentros: relatos de práticas desenvolvidas no Brasil: I curso de Especialização em gestão de hemocentros: resumos das monografias finais/ Ministério da Saúde, Secretaria de Atenção à Saúde, Departamento de Atenção Especializada. - Brasília: Ministério da Saúde, 2010.

PASSOS, M. C. P. Clima Organizacional do Hemolacen - Hemocentro de Sergipe: enfoque no Corpo Técnico da Diretoria de Atividades Hemoterapicas. In: Departamento de Atenção Especializada. Gestão de hemocentros: relatos de práticas desenvolvidas no Brasil: I curso de Especialização em gestão de hemocentros: resumos das monografias finais/ Ministério da Saúde, Secretaria de Atenção à Saúde, Departamento de Atenção Especializada. - Brasília: Ministério da Saúde, 2010.

PHOENIX LUFERCO. Phoenix Luferco ${ }^{\circledR}$. Empresa que comercializava o visor de hemaglutinação. Disponível em: <http://www.phoenix.ind.br/index.php?pgid=0>. Acesso em: 17 ago. 2018.

ROSA, M. F. L. Clima Organizacional do Hemocentro Coordenador do Estado do Acre Hemoacre. In: Gestão de hemocentros: relatos de práticas desenvolvidas no Brasil: I curso de Especialização em gestão de hemocentros: resumos das monografias finais/ Ministério da Saúde, Secretaria de Atenção à Saúde, Departamento de Atenção Especializada. - Brasília: Ministério da Saúde, 2010.

VIEIRA, N. U.; UNIVALI. Alunos criam protótipos de equipamento usado em análise sanguínea. 2016. Apresenta notícia sobre protótipos de visor de hemaglutinação. Disponível em: <https://www.univali.br/noticias/Paginas/alunos-criam-prototipos-deequipamento-usado-em-analise-sanguinea.aspx>. Acesso em: 17 out. 2017.

WHO (WORLD HEALTH ORGANIZATION). Guide to the selection and procurement of equipment and accessories. Department of Blood Safety and Clinical Technology, nov. 2002. 